\title{
Android-based automatic detection and measurement system of highway billboard for tax calculation in indonesia
}

\author{
Romi Fadillah Rahmat ${ }^{1}$, Sarah Purnamawati ${ }^{2}$, Handra Saito ${ }^{3}$, Muhammad Fariz Ichwan ${ }^{4}$, \\ Tri Murti Lubis ${ }^{5}$ \\ ${ }^{1,2,3,4}$ Department of Information Technology, Universitas Sumatera Utara, Indonesia \\ ${ }^{5}$ Department of Law, Universitas Sumatera Utara, Indonesia
}

\section{Article Info}

Article history:

Received Sep 28, 2018

Revised Nov 26, 2018

Accepted Dec 10, 2018

Keywords:

Android

Billboard detection

Billboard tax

Digital image processing

Perspective projection

\begin{abstract}
Billboards are objects, tools or actions, which based on the characteristics serve its own purpose to earn profits, advertise certain people or service, and to draw public's attention by placing it in a very strategic place. It has led the government to charge tax on billboards based on its location, dimensions, and viewpoints. Therefore, authorized parties have to be able to ensure the data authenticity of the proposed billboards. One of the obstacles in data verification is the time of billboards measurement process due to its size and height from the ground, based on this problem, and we developed a system which can measure the dimensions of billboards without physically touching it by implementing image processing methods to identify the billboards. The implementation is by measuring the dimensions of the billboards using perspective concept, then calculates the distance between the camera and the object using two-point distance calculation GPS coordinates. The results showed that the distance calculation using the GPS method generated inaccurate values, whereas the systematic distance method generated a result of errors' range from 0.5 to $25 \mathrm{~cm}$ if the image acquisition is performed nearly perpendicular to the object.
\end{abstract}

Copyright $\odot 2019$ Institute of Advanced Engineering and Science. All rights reserved.

\section{Corresponding Author:}

Romi Fadillah Rahmat,

Department of Information Technology,

Universitas Sumatera Utara,

Jl. Dr. T. Mansur No. 9, Medan 20155, North Sumatra, Indonesia.

Email: romi.fadillah@usu.ac.id

\section{INTRODUCTION}

Billboards are objects, tools or actions, which based on the characteristics serve its own purpose to earn profits, advertise certain people or service, and to draw public's attention by placing it in a very strategic place [1]. Billboards can be displayed in various types, shapes, and dimensions such as megatrons, Videotron, posters, banners, mobile, plaques, etc.

Nowadays, the role of billboards has become an effective communication system, not only for the manufacturers but also for the consumer. The capabilities of billboards and other promotional tools in delivering the message to consumers lead them to play essential roles in the success of their company in promoting its products and services.

The growing number of billboards has led the government to charge taxes to billboards based on their locations, dimensions, and viewpoints. However, before they are able to measure the billboards, they need to obtain the authorization from certain parties to ensure the authenticity of certain billboards. Furthermore, due to the large size of it, the government find it hard and dangerous to physically measure certain billboards because their tools are not long enough, this is where this system can be implemented as an alternative in measuring the billboards. 
There are many pieces of research on object calculation without measurement instrument using a camera or smartphone. However, most of their methods used are either auxiliary or inefficient. One of them is the measurement of object distance to camera and object dimension using accelerometer on a smartphone by shifting the smartphone according to the length and width of the object to find the object size and to shift from the starting point of the smartphone towards the object to calculate the distance [4]. Also, there is research where the calculation of distance and object size is calculated using the stereo vision image capture, and the distance of an object's size is measured by comparing the width and height of objects obtained from two images from two different cameras [5].

There is research that uses a more efficient way in which the measurement process uses only one camera from a smartphone which then is implemented to calculate the height of a tree using a proportional transformation. Where the trees are labeled with two markers to solve the measurement process [6]. Other research uses the formula to find the original height of an object with parameters such as the height of the object on the image, the height of the sensor, the focal length, the image height, and the distance of the object to the camera [7].

The taxation fee on billboards is based on its locations, dimensions, and several points of view. Authorized parties must ensure the data authenticity of the proposed billboards [11]. One of the obstacles in data verification is when the size of the billboards is very big, and the height from the ground is considerable. So that a system which has the ability to measure the dimensions of the billboards without measuring instruments is required.

\subsection{Billboard Tax}

The regions' administration offices issue the taxation of billboards and the imposition of billboard tax is not absolute in all regencies or cities in Indonesia. The administration office could choose whether to impose the tax to billboards or not. To be collected in a regency or municipality area, the regional government must first issue a local regulation concerning billboard tax which shall become the operational legal basis in the technical implementation of positioning and collecting billboard tax [2]. The formula to calculate the billboard tax is based on the regional regulation of Medan city no. 2 of 2004 [3] as follows.

$$
\operatorname{Tax}=T V+(S V * 25 \%)
$$

Where TV (Taxable Value) is the result of multiplication between the area of a billboard, the installation period and the rental value of the billboard, SV (Strategic value) represents a value that set at the installation location's point, and $25 \%$ is the official billboard tax fee.

\subsection{Perspective Projection}

Perspective projection is a 3-dimensional mapping which is converted into 2 dimensions. Moreover, it has the same principle with the way human vision and the camera works.

From Figure 1, there is a standard formula in searching for a large angle of Q. The formula for the large angle of $\mathrm{Q}$ can be seen in the equations below.

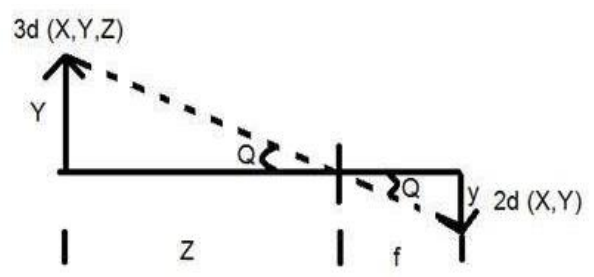

Figure 1. Projection of 3D object into a 2D object

$$
\begin{aligned}
& \operatorname{Tan} Q=\frac{\mathrm{Y}}{\mathrm{Z}} \\
& \operatorname{Tan} Q=\frac{\mathrm{Y}}{\mathrm{f}}
\end{aligned}
$$


Where $\mathrm{Y}$ is the original height of the object, $\mathrm{Z}$ is the distance between camera and object, $\mathrm{y}$ represents the object height on camera and $\mathrm{f}$ is the focal length on camera. The combination of both equations above creates the following equation due to the same size of the angle.

$$
\frac{\mathrm{Y}}{Z}=\frac{\mathrm{y}}{\mathrm{f}}
$$

Based on the previous equation, the original height of an object can be found by using the former equation below and vice versa with the width of an object using the latter equation where $\mathrm{W}$ is the original width of the object and w is object's width on images.

$$
\begin{aligned}
& Y=\frac{y \cdot Z}{f} \\
& W=\frac{w \cdot Z}{f}
\end{aligned}
$$

The required variables such as the height of the object on the camera in pixel size, the distance between the camera and the object, also the camera's focal length, the original height of the object can be found using the latter equation above.

\section{RESEARCH METHOD}

The proposed method for identifying the dimension of a billboard by the image consists of several steps which are: Determining the latitude and longitude point based on GPS in the smartphone. Frame per frame retrieval from smartphone camera; Gray-scaling; Noise reduction using Gaussian blur [8]. Edge detection using Canny edge method[10], then eliminate small blobs with erosion and dilation. Contour search and tagging that have the four most substantial angles and widths, then change the position of the object perpendicular to the camera with perspective transform. Determine the latitude and longitude and calculate the distance between both latitude and longitude point. Search the length and width of the billboard by using physics optical formula. After those steps are done based on the obtained length and width of billboards can calculate the total billboard tax to be imposed by the government. Detailed explanations of the steps undertaken will be explained in the following sections. The general architecture that describes the method in this research is shown in Figure 2.

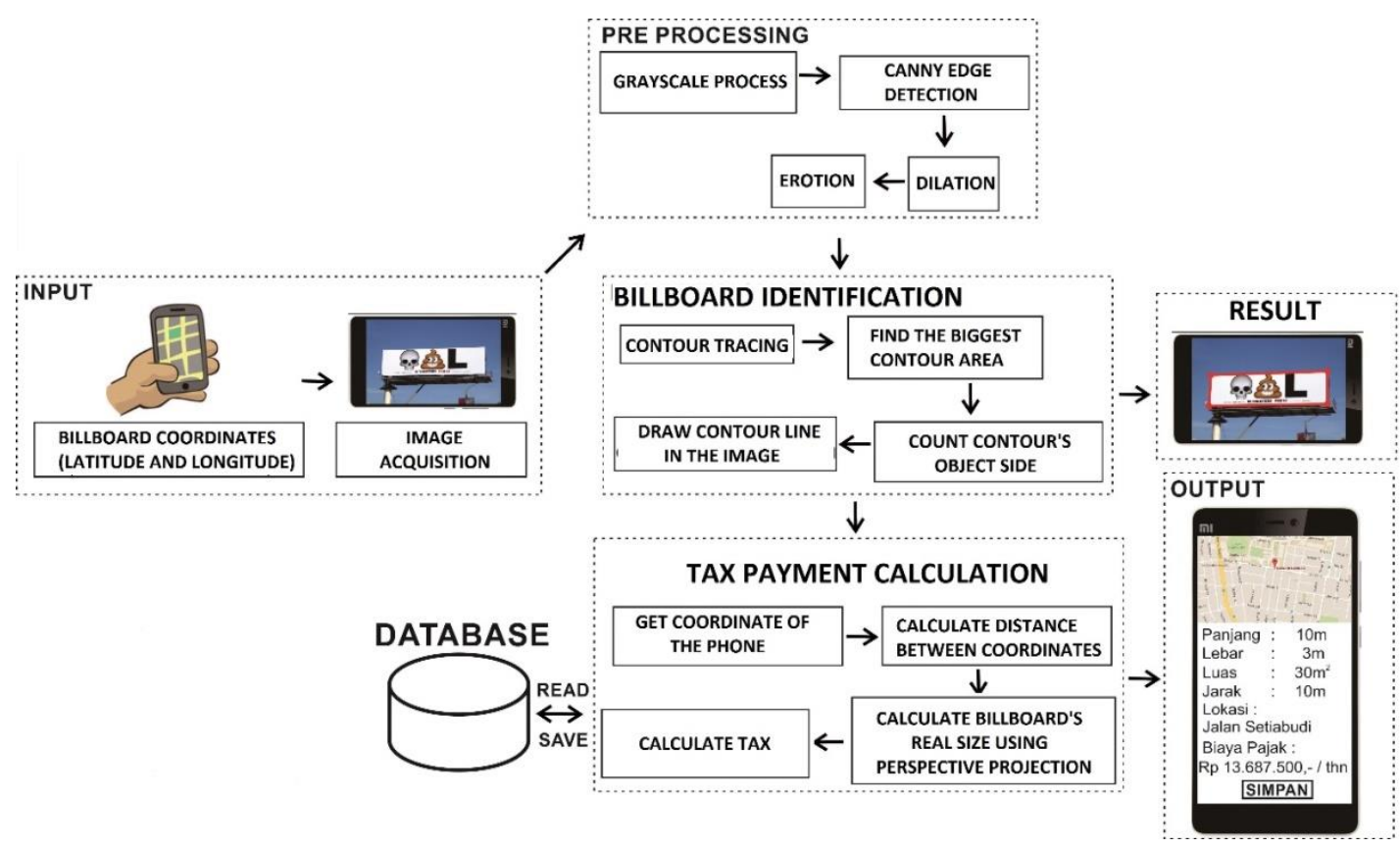

Figure 2. General architecture 


\subsection{Data Acquisition}

The data that used in this study are frame by frame image data which collected in real-time mode. The image is taken by Android smartphone. Several factors which give impact to the quality of the image are camera's quality, environment lightning, the object in front of the billboard, the range between camera and billboard, and the angle of the camera to the billboard. For this process, we took 30 billboard image and using Caterpillar Handphone with GPS and A-GPS inside, authors also used Garmin GPS to increase our location accuracy. While in term of the image we took it using 13 megapixels camera with 1080x1920 pixel resolution.

\subsection{Determination of Latitude and Longitude Point}

Latitude and longitude point can be implemented directly using the available code on Android programming library which is LocationManager class. At first, LocationUpdate function request will be imported to get the information of the smartphone's location, then calling the getLastKnowLocation function to retrieve the object from Location class where the latitude can be obtained by calling the getLatitude function and the longitude point by calling the getLongitude function.

\subsection{Pre-processing}

Before going to the next phase which is the identification of the image, the image has to go through several steps in the pre-processing which is gray-scaling, Canny edge detection, image morphology process which consists of two sub-process (dilation and erosion). In this pre-process we used the OpenCV library for image processing. This particular library is purposed to do image processing in video frames in realtime mode. It consists of 400 functions and ranging in 28 areas of image processing and analysis [9].

\subsubsection{Grayscaling}

Frame (image) that was obtained from the camera will be converted into a grayscale image. In OpenCV, there is a function to convert the RGB image to grayscale image, i.e., cvtColor (), or it can be shown in the formula below [12]:

$$
G=0.299(R)+0.558(G)+0.114(B)
$$

The result of the conversion of RGB image value to grayscale image value can be seen in Figure 3 .
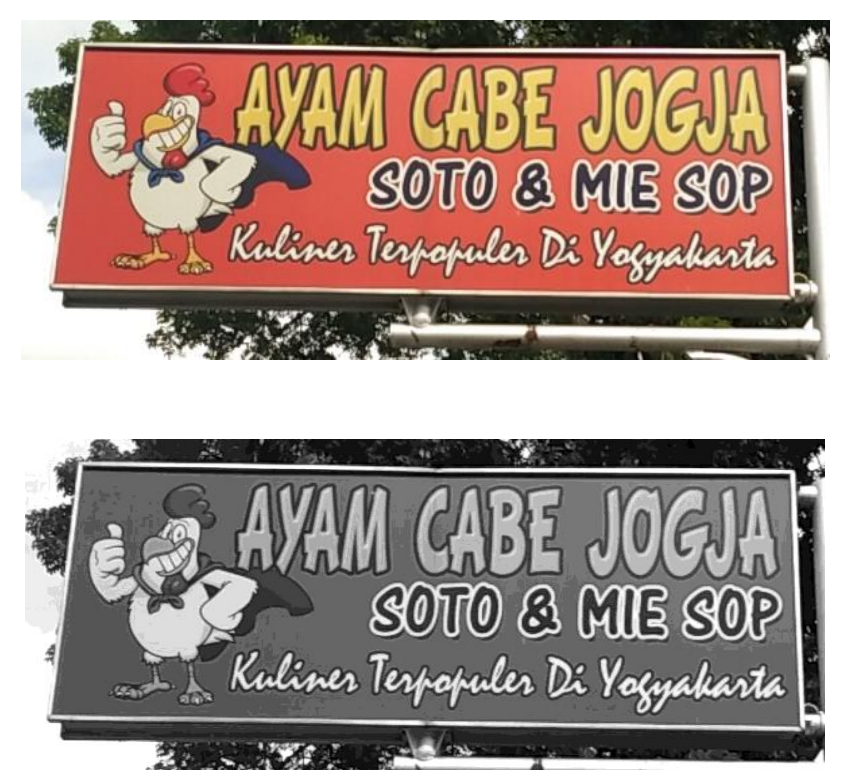

Figure 3. Conversion process of RCB image to a grayscale image

\subsubsection{Canny Edge Detection}

After removing noise on an image, the next phase is edge detection using Canny Edge Detector method. The algorithm is as follows: 
a) Implement noise reduction with $5 \times 5$ Gaussian Filter using this equation below :

$$
\begin{aligned}
& H_{i j}=\frac{1}{2 \pi \sigma^{2}} \exp \left(-\frac{(i-(k+1))^{2}+(j-(k+1))^{2}}{2 \sigma^{2}}\right) \\
& 1 \leq i, j \leq(2 k+1)
\end{aligned}
$$

b) Apply Sobel kernel in horizontal and vertical perspective and get its first derivative. By those two images we can find the edge gradient and the direction for each resulted pixel as follows:

$$
\begin{aligned}
& m(\text { gradient })=\sqrt{G_{x}^{2}+G_{y}^{2}} \\
& \theta=\arctan \left(\frac{G_{y}}{G_{x}}\right)
\end{aligned}
$$

c) Scan all the image pixel to remove unnecessary pixel which is not related to the edge by using nonmaximum suppression. After that, we will get edges with a thin line.

d) Do Hysteresis Thresholding, which has a purpose of reconnecting the broken thin edges produced in the previous steps.

In OpenCV, the function to do Canny Edge Detection is Canny(). The explanation of the Canny() function is given as follows.

Canny(src, dst, thres1, thres2, apertureSize)

The parameters are $s r c$ for grayscale input image, $d s t$ for binary output image with a detected edge, thres 1 represents the initial limit value for hysteresis procedure, thres 2 is the final limit values for hysteresis procedure and apertureSize as the integer value to determine the matrix dimension on Sobel operator. The result of edge detection from a grayscale image using a canny method can be seen in Figure 4 .

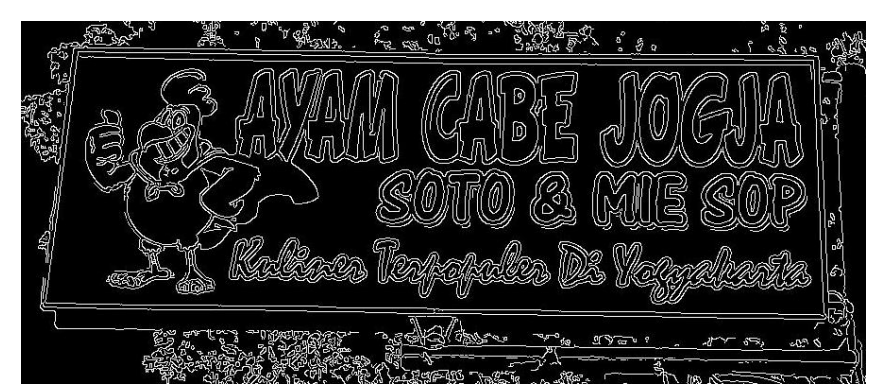

Figure 4. Canny image detection image

\subsubsection{Dilation and Erosion}

The generated edges from canny edge detection are still in dashed and undesirable edge line remained. Therefore, dilation and erosion are required to combine all the dashed edges and to eliminate small lines that are considered as edges. The functions for dilation and erosion in OpenCV are dilate() and erode() respectively. The erode() and dilate() functions have the same parameter yet give different output. The erode () and dilate () functions can be described as follows:

Dilate(src, dst, kernelSize)

Erode(src, dst, kernelSize)

The parameters are $s r c$ as the binary input image, $d s t$ for the dilated or eroded output image and kernelSize represents the determination of matrix dimension used for dilation or erosion process. The default value for kernel size is $3 \times 3$. The resulting image of the dilation and erosion process can be seen in Figure 5. 

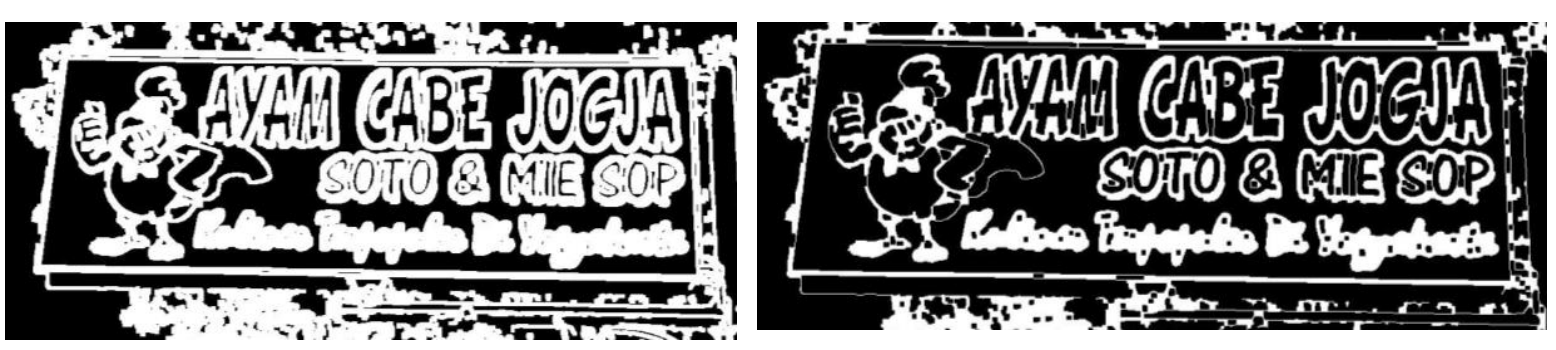

Figure 5. Erosion and dilation images

\subsection{Billboard Identification}

This stage is the processes where the application can identify the billboard object to be tested.

\subsubsection{Contour Tracing}

In the contour tracing step, edge line that forms a plane (contour) will be stored the. OpenCV provides a function to find the contour which is findContour() that produce identified contour points as the output. The identified points can be illustrated using drawContour() function. The findContour() and drawContour()functions can be described as follows:

findContour(src, contours, hierarchy, mode, method)

The parameters are $s r c$ as the binary input image, contours represents the identified contour coordinate point will be stored in a vector, hierarchy is the optional output vector contained information about image topology, mode for the contour points detection which is using Imgproc.RETR_LIST for this research. While method is the contour approaching method In this research using Imgproc.CHAIN_APPROX_NONE that obtain and store all the contour points. The image of a contour detection result can be seen in Figure 6.

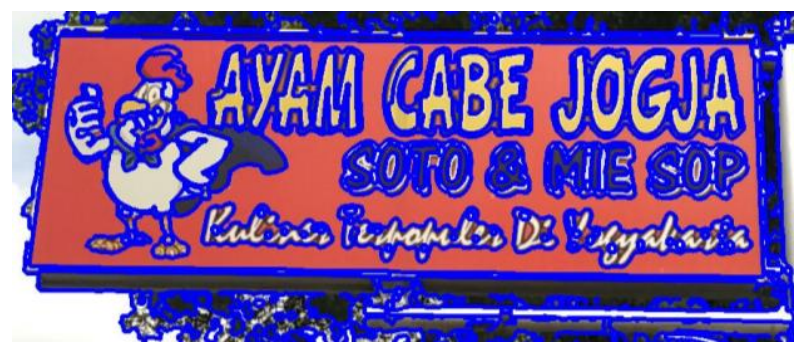

Figure 6. Identified contour image

\subsubsection{Filter Contour}

After the contour is obtained, a contour that does not match the desired image detection is still available. Therefore, it is necessary to process the filter on the contour by searching the most significant area and looking for some angles or curves contained in each contour so that the contour obtained is the billboard that wants to be measured. Function in OpenCV to calculate the contour area is contourArea() and the one to find the angle number is approxPolyDP( $)$. The filter process is done because the billboards must have the largest contour area and rectangular shape or have four angles on the image so that the other contour will be eliminated. The following explanation of the function described as follows.

contourArea(contour)

Parameter :

Contour : Contour point to which the value of the area is calculated.

approxPolyDp(contour, approxCurve, epsilon, closed) 
The parameters are contour as the contour point input, approxCurve for the output in the form of coordinates point obtained from the angles, epsilon is the double value that determines the value of accuracy in finding angles and closed represents the boolean value that determines whether the angles are connected or not. The image of the contour filtering process can be seen in Figure 7.

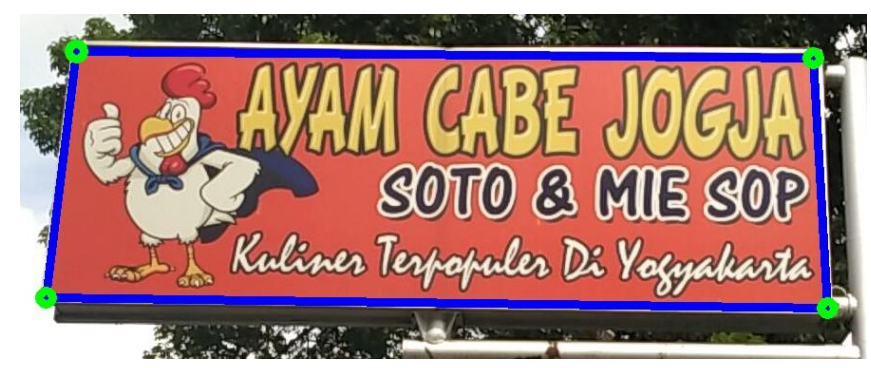

Figure 7. Image of the contour filtering process

\subsubsection{Perspective Settings}

Billboards object are not entirely rectangular because the shots are not always precisely perpendicular to the billboards. Therefore, minAreaRect() function is used in OpenCV with billboard contour that obtained from contour filtering as the parameter to find a rectangular shape that matches the shape of the billboard caught on camera. The rectangularly shaped result of minAreaRect() can be seen in Figure 8 .

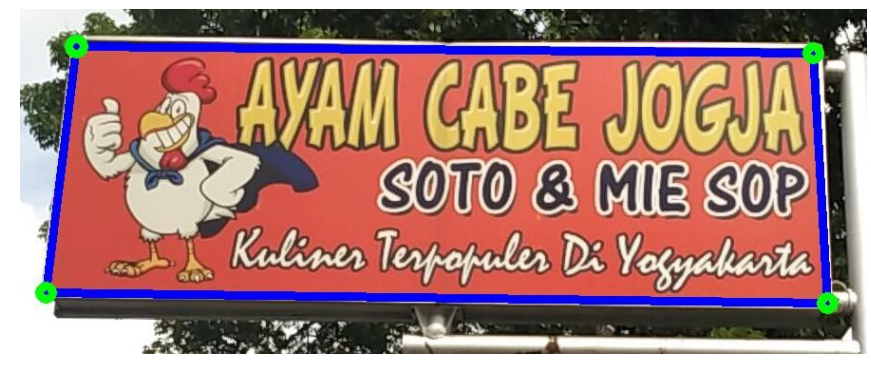

Figure 8. Result of minAreaRect() function

\subsection{Calculation of Billboards Dimension and Tax Charges \\ 2.5.1 Calculation of Billboard Dimension}

The calculation of billboards dimension can be calculated using perspective projection concept. The width and height of the object in the image were obtained from rectangular shaped by minAreaRect() function. In this research, the focal length has a default value which is 1740 . Suppose that the width of the object in the image is 652 pixels, the height of is 392 pixels and the object distance to the camera is $400 \mathrm{~cm}$, then the original width and height of the object can be calculated as follows:

$$
\begin{array}{ll}
Y=\frac{392 * 400}{1740} & W=\frac{652 * 400}{1740} \\
Y=\frac{156800}{1740} & W=\frac{260800}{1740} \\
Y=90,11 \mathrm{~cm} & W=149,89 \mathrm{~cm}
\end{array}
$$

\subsubsection{Calculation of Tax Charges}

Billboard tax charges can be calculated using equation (7). Suppose the sample billboard in Setia Budi Rd, which is an elite area, then its rental value $\mathrm{Rp} 9.000$,- and its strategic value at $\mathrm{Rp} 30.000 .000,-$. Therefore, the tax charges that need to be paid can be calculated as follows.

$$
\begin{gathered}
\operatorname{Tax}=\left(\left(\frac{149,89 * 90,11}{10.000}\right) * 365 * 9.000\right)+(30.000 .000 * 0.25) \\
\operatorname{Tax}=4.436 .914,12515+7.500 .000 \\
\operatorname{Tax}=R p 11.936 .914,12515 \text { anually }
\end{gathered}
$$


As the tax charge calculation finish, then the data will be inputted into the system, which will be used to be compared in another inspection. This is to solve the problem that sometimes the size of the billboard and the tax calculation which was filled inside the tax paper is written differently from its actual size in the field.

\section{RESULTS AND ANALYSIS}

In this section, we described the result of the system based on the error or difference between its original size and predicted size by the system. We also calculate the accuracy of the system, using 30 different billboards of different size and location.

Before we proceed, Figure 9 and Figure 10 shows the final interface before the data send to the server. Figure 9 shows the final capture of the billboard, wherein this stage we already have the billboard measurement.

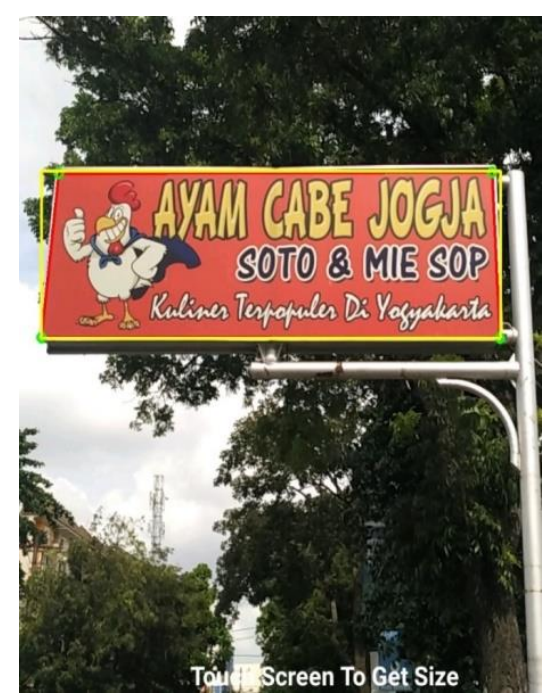

Figure 9. Final capture to get size

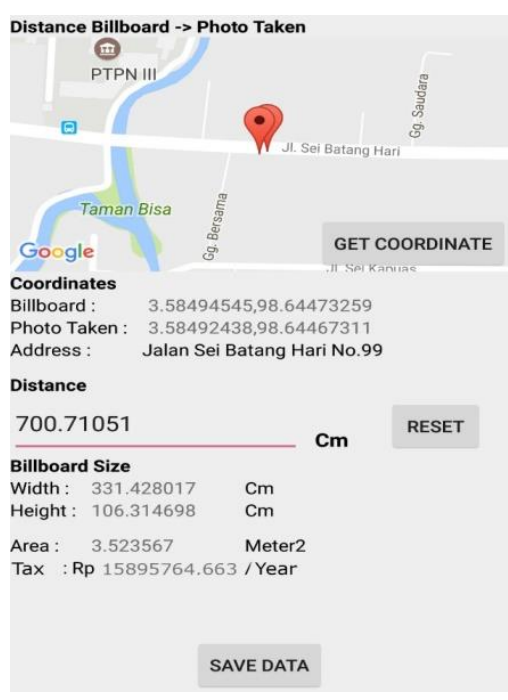

Figure 10. Tax calculation

In Figure 10 we took two coordinates based on two different location, they are a billboard location, and the photo was taken location. These two location is used to measure distance so that we can get the real size of the billboard, if the GPS is not working correctly we can use the column for manual distance based on estimation. Once we get the generated data, it will be saved and send to the server.

Based on the results in Table 1, we can see that the between billboard and image acquisition plays an important part. The distance usually in 5-20 meters between those two points. The distance will contribute to the angle of the image. We have to consider to take billboard image as flat as possible.

We can see that based on the table there is a different error in terms of width and height of the image if the billboard's size larger than $300 \mathrm{~cm}$, billboard no 4, 12, 21, 22, has error value of $\pm 10-20 \mathrm{~cm}$, which will affect tax calculation from the system. However, in the real-life application, the tax calculation and size measurement of the billboard from our system is considered estimation and tools for a legal decision from the right authority.

If we assume the lousy result that has error distance value is larger than $15 \mathrm{~cm}$, we can say that out of 30 testing images; we will have 25 good results and five bad result, which means we get $83,3 \%$ accuracy value. However, it is still acceptable in the real-life implementation, because usually, the tax authority will classify the size of the billboard into a particular category.

The GPS accuracy plays an essential role to measure the correct size measurement. The dependencies of an accurate device is a must. GPS in a standard smartphone sometimes will give lousy result also. However, it usually gives a problem with the first data taken, while once recorded in the system it can be considered for the next data taken. Aside from that, image processing in the system gives good accuracy. However, improvement is a must. Some of the billboard images have noise because of other objects in front of the billboard, such as cable, leaves, tree, or another billboard object. This problem will become our next research, which we assume can be solved if we add machine learning to the system. 
Table 1. Pre-processing Result

\begin{tabular}{|c|c|c|c|c|c|c|}
\hline No & Width $(\mathrm{cm})$ & Height $(\mathrm{cm})$ & Predicted Width $(\mathrm{cm})$ & Predicted Height $(\mathrm{cm})$ & Width Error $(\mathrm{cm})$ & Height Error $(\mathrm{cm})$ \\
\hline 1 & 240 & 50 & 247.6552 & 51.4942 & -7.6552 & -1.4942 \\
\hline 3 & 140 & 50 & 137.6034 & 48.6437 & $+2,966$ & $+1,3563$ \\
\hline 5 & 120 & 80 & 122.3621 & 74.0115 & $-2,3621$ & $+5,9885$ \\
\hline 6 & 400 & 130 & 402.0402 & 128.9655 & $-2,0402$ & $+1,0345$ \\
\hline 7 & 150 & 150 & 152.3403 & 151.0938 & +2.3403 & +1.0938 \\
\hline 10 & 300 & 200 & 303.0393 & 198.0382 & +3.0393 & -1.9618 \\
\hline 11 & 350 & 400 & 359.0545 & 415.8374 & +9.054 & +15.8374 \\
\hline 12 & 450 & 200 & 477.7232 & 205.9384 & +27.7232 & +5.9384 \\
\hline 13 & 200 & 200 & 203.6734 & 194.2311 & +3.6734 & $-5,7689$ \\
\hline 14 & 240 & 220 & 240.9853 & 203.2182 & +0.9853 & $-16,7818$ \\
\hline 19 & 300 & 150 & 291.8115 & 148.0517 & $-8,1885$ & $-1,9483$ \\
\hline 20 & 400 & 250 & 400.6584 & 255.3238 & +0.6584 & +5.3238 \\
\hline 21 & 450 & 250 & 472.8374 & 253.4933 & +22.8374 & +3.4933 \\
\hline 22 & 400 & 250 & 423.2329 & 252.3233 & +23.2329 & +2.3233 \\
\hline 23 & 300 & 200 & 307.8943 & 212.6832 & +7.8943 & +12.6832 \\
\hline 24 & 200 & 150 & 205.0023 & 153.3948 & +5.0023 & +3.3948 \\
\hline 25 & 150 & 90 & 152.9384 & 93.4834 & +2.9384 & +3.4834 \\
\hline 26 & 300 & 150 & 301.9383 & 150.2649 & +1.9383 & +0.2649 \\
\hline 27 & 400 & 100 & 415.6254 & 100.3495 & +15.6254 & +0.3495 \\
\hline 28 & 240 & 100 & 248.3845 & 107.0845 & +8.3845 & +7.0845 \\
\hline 29 & 250 & 150 & 250.4383 & 155.3948 & +0.4383 & +5.3948 \\
\hline 30 & 140 & 50 & 147.5323 & 52.2356 & +7.5323 & +2.2356 \\
\hline
\end{tabular}

\section{CONCLUSION}

Following the research and its experimental activities conducted, it is concluded that:

a) GPS plays an essential part as a reference in finding distances due to its inefficiency and inaccuracy in obtaining the distance point.

b) The calculation of the billboard's size can be done using perspective projection concepts, as long as the image taken by the user is perpendicular to the billboard.

c) The recognition of billboards using image processing is strongly opposed due to many factors such as lighting, the surrounding environment, a contrast of billboards color with its surrounding environment, and the placement of billboards that make the edge detection unsuitable as desired, resulting in unrecognized billboards.

Based on the research conducted, the suggestions that expected to do in further research as follows:

a) Use accurate GPS device so the measurement will perform well.

b) In image processing we have several issues in the future, they measure the billboard regardless of the angle of the captured image, eliminates other noise, implementing other detailed segmentation to get different and better result.

c) Implementing machine learning to recognize the billboard image.

\section{REFERENCES}

[1] R. Burton, Taxation. Jakarta: STIE Perbanas, 1998.

[2] S. W. Hulu, Basis for Imposition of Billboard Tax In Medan City Parks Department, Medan: Universitas Sumatera Utara, 2012.

[3] Major, Medan City Regional Regulation No. 2 of 2004: Indonesian Billboard Tax.

[4] S. Laotrakunchai, A. Wongkaew, and K. Patanukhom, "Measurement of Size and Distance of Objects Using Mobile Devices," in Int. Conf. on Signal-Image Technology \& Internet-Based Systems, pp. 157-161, 2015.

[5] Y. M. Mustafah, R. Noor, H. Hasbi, and A. W. Azma, "Stereo Vision Images Processing for Real-time Object Distance and Size Measurements," in Int. Conf. on Computer and Communication Engineering, pp. 659-663, 2012.

[6] D.Han, and C. Wang, "Tree Height Measurement based on image processing Embedded in Smart Mobile phone," in Int. Conf. on Multimedia Technology, pp. 3293-3296, 2011.

[7] A. N. Murali, "Machine Vision for General Cameras for Quality Testing and Dimension Calculations," in Int. J of Modeling and Optimization, vol. 5, no. 4, pp. 295 - 299, 2015.

[8] Bozkurt, F., Yağanoğlu, M., \& Günay, F. B. Effective Gaussian Blurring Process on Graphics Processing Unit with CUDA. International Journal of Machine Learning and Computing, vol. 5, no. 1, pp: 57 - 61, 2015. 
[9] Ionel, S., Alexandru, I., \& Catalin, M. Algorithms for Objects' Shape Recognition and Measurement before Gripping. The Annals of "Dunarea de Jos" University of Galati, vol. 1, no. 2, pp: 11 - 16, 2011.

[10] Nisha, Mehra, R., \& Sharma, L. Comparative Analysis of Canny and Prewitt Edge Detection Techniques used in Image Processing. International Journal of Engineering Trends and Technology (IJETT), vol. 28, no. 1, pp: 48-53, 2015.

[11] Sunarimahingsih, Y. T et al. The significance of Billboard in Semarang City, Scientific journal, vol. 15, no. 1, pp: $44-58,2013$.

[12] R. F. Rahmat, T. Chairunnisa, D. Gunawan, and O. S. Sitompul, "Skin color segmentation using multi-color space threshold," in 3rd International Conference on Computer and Information Sciences, ICCOINS 2016 - Proceedings, pp. 391-396, 2016.

\section{BIOGRAPHIES OF AUTHORS}

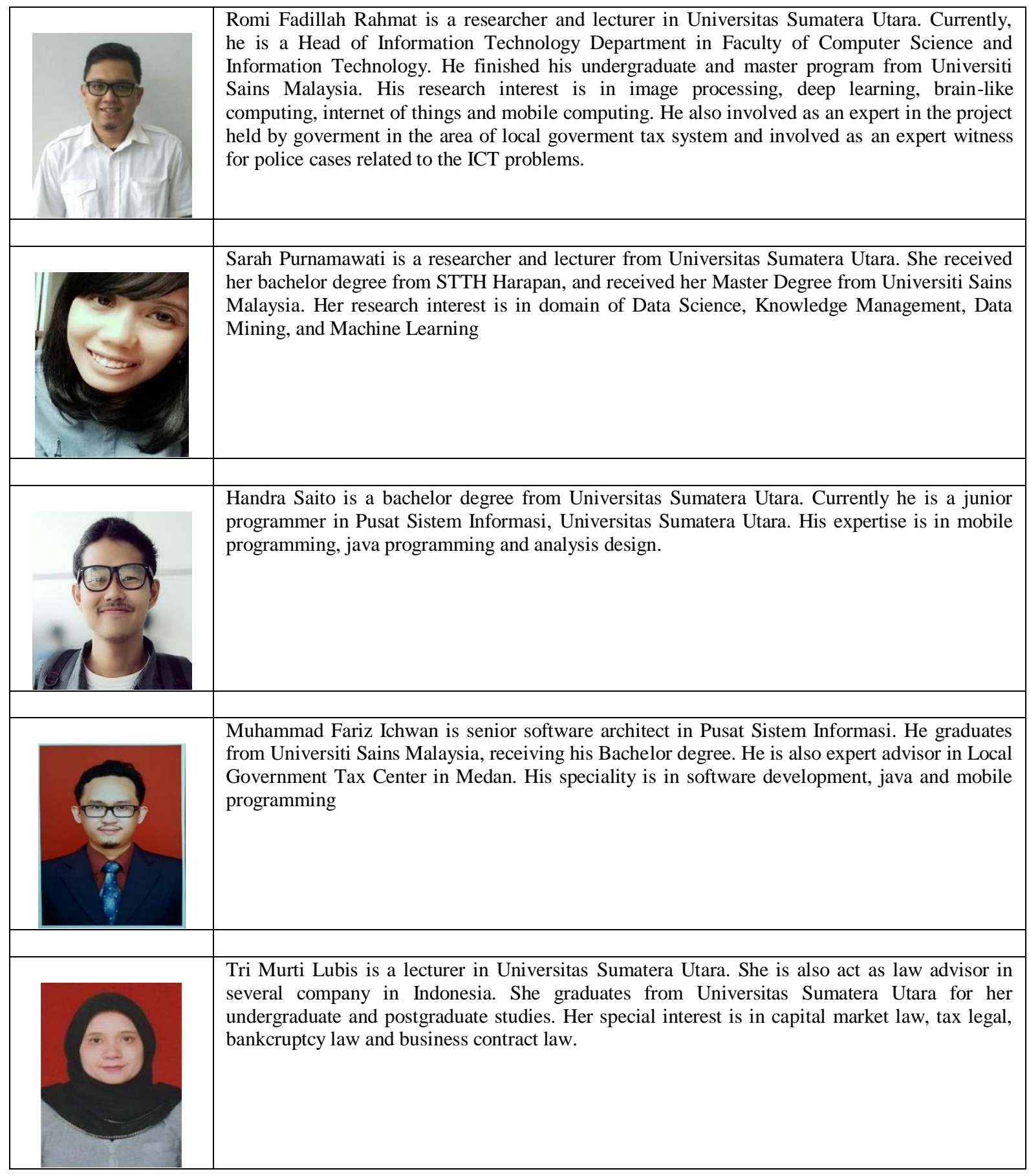

